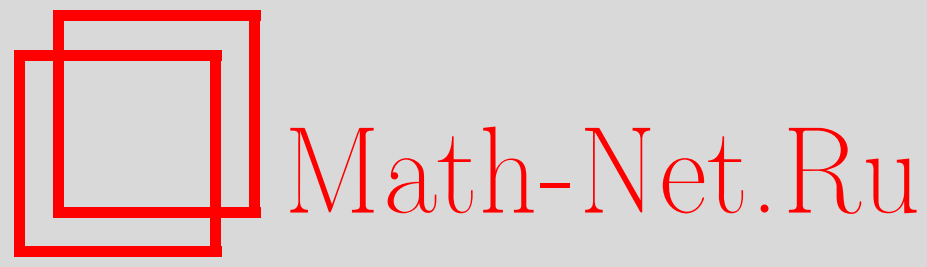

Джань-Дун Мэй, Цзи-Гэнь Пэн, Корректность одного класса популяционных уравнений с зависимостью от возраста, Функи. анализ и его прил., 2015, том 49, выпуск 2, 92-96

DOI: https://doi.org/10.4213/faa3197

Использование Общероссийского математического портала MathNet.Ru подразумевает, что вы прочитали и согласны с пользовательским соглашением http://www . mathnet.ru/rus/agreement

Параметры загрузки:

IP : 54.237 .206 .68

26 апреля 2023 г., 12:29:08

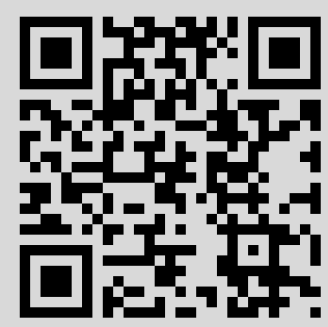


[5] A. Malchiodi, Boll. Unione Mat. Ital., Ser. B, 8:3 (2005), 615-628. [6] N. Mizoguchi, T. Suzuki, Houston J. Math., 22:1 (1996), 199-215. [7] А. И. Назаров, Труды С.-Петербург. MO, 10 (2004), 33-62. [8] А. И. Назаров, Пробл. матем. анализа, 20 (2000), 171-190.

Санкт-Петербургский государственный университет e-mail: sergey.kolonitskii@gmail.com

Поступила в редакцию 21 января 2014 г.

УДК 517.9

\title{
Корректность для одного класса популяционных уравнений с зависимостью от возраста*
}

\author{
(c) 2015. ДжАнь-Дун Мэй, Цзи-Гэнь Пэн
}

1. Введение. Рассмотрим следующую задачу для популяционного уравнения с зависимостью от возраста и запаздыванием, описывающего процесс рождения и смерти с учетом смерти по причине беременности:

$$
\left\{\begin{array}{l}
\frac{\partial x(t, a)}{\partial t}=-\frac{\partial x(t, a)}{\partial a}-\mu(a) x(t, a)-\alpha(a) x(t-r, a), \\
x(t, 0)=\int_{0}^{\infty} \int_{-r}^{0} \beta(\sigma, a) x(t+\sigma, a) d \sigma d a, \quad t \geqslant 0, \\
x(s, a)=q(s, a), \quad s \in[-r, 0], a \geqslant 0,
\end{array}\right.
$$

где $r>0,0 \leqslant \mu, \alpha \in L^{\infty}\left(\mathbb{R}^{+}\right), \beta \in L^{\infty}\left([-r, 0] \times \mathbb{R}^{+}\right)$. Это уравнение представляет плотность населения возраста $a$ в момент $t$. Функции $\mu$ и $\alpha-$ это смертность по естественным причинам и смертность по причине беременности соответственно, а $\beta$ - коэффициент рождаемости. Уравнение

$$
x(t, 0)=: B(t)=\int_{0}^{\infty} \int_{-r}^{0} \beta(\sigma, a) x(t+\sigma, a) d \sigma d a, \quad t \geqslant 0,
$$

называется процессом рождения.

Для случая, когда $\alpha=0$, такие модели были введены Лоткой в 1925 г. и изучались многими авторами. В [2] и [8] к популяционным уравнениям успешно применялась теория полугрупп. В частности, в [2] систематически изучено асимптотическое поведения решения задачи (1) для случая, когда процесс рождения задан соотношением $B(t)=\int_{0}^{\infty} \beta(a) x(t, a) d a, t \geqslant 0$, где $\beta$ - коэффициент рождаемости. В недавних работах [6] и [7] доказано, что задача (1) корректна при $\alpha=0$, и изучены асимптотические свойства ее решения.

* Работа выполнена при поддержке Фонда естественных наук Китая (гранты 11301412 и 11131006), Исследовательского фонда для докторских программ высших школ Китая (грант 20130201120053), Фонда естественных наук провинции Шаньси Китая (грант 2014JQ1017), проекта Постдокторального научного фонда Китая (2014M550482) и Фонда фундаментальных исследований для центральных университетов (контракт 2012jdhz52). Часть работы была выполнена во время визита первого автора к профессору Биргит Якоб в Вуппертальский университет, Германия. 
Однако в практических приложениях $\alpha$ не может быть нулевой, поскольку имеет место смертность по причине беременности. K сожалению, к настоящему времени авторам не известна ни одна работа, в которой доказывалась бы корректность соответствующей задачи. Цель настоящей заметки - доказать эту корректность. Нашим основным средством является теорема об инвариантности допустимости, установленная в нашей недавней работе [5]. Для этого нам надо ввести некоторые основополагающие понятия.

Пусть $1<p<\infty$, а $X, U$ и $Y$ - банаховы пространства. Корректной линейной системой на $(X, U, Y)$ в смысле Саламона [9] и Вейса [12] называется такая четверка $\Sigma=\left(M, \Phi, \Psi_{\infty}, F_{\infty}\right)$, что

(i) $M=(M(t))_{t \geqslant 0}$ есть $C_{0}$-полугруппа, порождаемая оператором $K$ на $X$;

(ii) $\Phi=(\Phi(t))_{t \geqslant 0}$ - такое семейство ограниченных линейных операторов из $L^{p}\left(\mathbb{R}^{+}, U\right)$ в $X$, что $\Phi(t+\tau) u=M(t) \Phi(\tau) u+\Phi(t) u(\cdot+\tau)$ для любого $и$ из $L^{p}\left(\mathbb{R}^{+}, U\right)$ и любых неотрицательных $\tau$ и $t$;

(iii) $\Psi_{\infty}$ - такой непрерывный линейный оператор из $X$ в $L_{\text {loc }}^{p}\left(\mathbb{R}^{+}, Y\right)$, что $\left(\Psi_{\infty} x\right)(s)=\left(\Psi_{\infty} M(\tau) x\right)(s-\tau)$ для любого $x \in X$ и любых $s>\tau \geqslant 0$;

(iv) $F_{\infty}$ - такой непрерывный линейный оператор из $L^{p}\left(\mathbb{R}^{+}, U\right)$ в $L_{\mathrm{loc}}^{p}\left(\mathbb{R}^{+}, Y\right)$, что $\left(F_{\infty} u\right)(s)=\left(\Psi_{\infty} \Phi(\tau) u+F_{\infty} u(\cdot+\tau)\right)(s-\tau)$ для любого $u$ из $L^{p}\left(\mathbb{R}^{+}, U\right)$ и любых $s$ и $\tau$, таких, что $s>\tau \geqslant 0$.

Если пара $(M, \Phi)$ удовлетворяет условиям (i) и (ii), то она называется $а б$ страктной линейной системой управления на $(X, U)$. Каждой системе управления соответствует единственный оператор управления $\mathfrak{B} \in L\left(U, X_{-1}\right)$, удовлетворяющий условию $\int_{0}^{t} M_{-1}(t-s) \mathfrak{B} u(s) d s \in X$ для любого $u$ из $L^{p}\left(\mathbb{R}^{+}, U\right)$ и любого неотрицательного $t$ (он называется допустимым оператором управления); см. [10]. Здесь $M_{-1}=\left(M_{-1}(t)\right)_{t \geqslant 0}$ - экстраполяционная полугруппа. Для любого $t \geqslant 0$ оператор $M_{-1}(t)$ является непрерывным продолжением оператора $M(t)$ на экстраполяционное пространство $X_{-1}^{K}$, где $X_{-1}^{K}$ - пополнение пространства $X$ по норме $\left\|R\left(\lambda_{0}, K\right) \cdot\right\|$, а $R\left(\lambda_{0}, K\right)$ - резольвента оператора $K, \lambda_{0}$ - произвольный заданный элемент из резольвентного множества оператора $K$. Генератор полугруппы $M_{-1}$ является продолжением оператора $K$ на $X_{-1}^{K}$; обозначим указанный генератор через $K_{-1}$ (см. [1]). В этом случае будем говорить, что $(K, \mathfrak{B})$ порождает систему $(M, \Phi)$. Если $(M, \Psi \infty)$ удовлетворяет условиям (i) и (iii), то существует единственный оператор $\mathfrak{C}$ из $L(D(K), Y)$, удовлетворяющий неравенству $\int_{0}^{T}\|\mathfrak{C} M(t) x\|^{p} d t \leqslant c(T)\|x\|^{p}$ для любого $x$ из $D(K)$ и соотношению $\left(\Psi_{\infty} x\right)(t)=\mathfrak{C} M(t) x$ для любого $x$ из $D(M)$ и любого неотрицательного $t$; этот оператор называется допустимым оператором наблюдения (см. [11]). В этом случае мы также будем говорить, что $\left(M, \Psi_{\infty}\right)$ является абстрактной линейной системой наблюдения на $(X, Y)$, порождаемой парой $(K, \mathfrak{C})$. Корректная линейная система $\Sigma=\left(M, \Phi, \Psi_{\infty}, F_{\infty}\right)$ называется регулярной линейной системой, если $\lim _{s \rightarrow 0} \frac{1}{s} \int_{0}^{s}\left(F_{\infty} u_{0}\right)(\sigma) d \sigma=0$ в $Y$ при постоянной входной функции $u_{0}(t)=z, z \in U, t \geqslant 0$. Будем говорить, что тройка $(K, \mathfrak{B}, \mathfrak{C})$ порождает эту регулярную линейную систему. Нетрудно доказать, что тройка $(K, \mathfrak{B}, \mathfrak{C})$ порождает регулярную линейную систему тогда и только тогда, когда

(i) $K$ порождает $C_{0}$-полугруппу;

(ii) $\mathfrak{B}$ является допустимым для $K$;

(iii) $\mathfrak{C}$ является допустимым для $K$; 
(iv) $\Phi(t) u \in D\left(\mathfrak{C}_{\Lambda}^{K}\right)$ для любого $u$ из $L^{p}\left(\mathbb{R}^{+}, U\right)$ и п.в. $t \geqslant 0$, а $\mathfrak{C}_{\Lambda}^{K} \Phi \cdot \epsilon$ $L\left(L_{\text {loc }}^{p}\left(\mathbb{R}^{+}, U\right), L_{\text {loc }}^{p}\left(\mathbb{R}^{+}, Y\right)\right)$ (см. [12]). Здесь $\mathfrak{C}_{\Lambda}^{K} x=\lim _{t \rightarrow 0} \mathfrak{C} \lambda R(\lambda, K) x$, а $D\left(\mathfrak{C}_{\Lambda}^{K}\right)$ $=\left\{x \in X:\right.$ предел $\lim _{t \rightarrow 0} \mathfrak{C} \lambda R(\lambda, K) x$ существует $\}$.

Полугруппа левых сдвигов определяется на $L^{p}([-r, 0], X)$ следующим обра30M:

$$
\left(S_{U}(t) g\right)(\theta):= \begin{cases}g(t+\theta), & \text { если } t+\theta \leqslant 0, \\ 0 & \text { в противном случае }\end{cases}
$$

при $t \geqslant 0$ и $-r \leqslant \theta \leqslant 0$. Ее генератор определяется формулой $Q_{U}:=d / d \theta$; его область определения $D\left(Q_{U}\right)$ есть множество $\left\{g \in W^{1, p}([-r, 0], U): g(0)=0\right\}$. Рассмотрим оператор $\phi(t): L^{p}\left(\mathbb{R}^{+}, U\right) \rightarrow L^{p}([-r, 0], U)$, заданный формулой

$$
(\phi(t) u)(\theta)= \begin{cases}u(t+\theta), & \text { если } t+\theta \geqslant 0, \\ 0 & \text { в противном случае }\end{cases}
$$

при $t \geqslant 0$ и $-1 \leqslant \theta \leqslant 0$. Хадд доказал, что $\left(S_{U}, \phi\right)$ есть абстрактная линейная система управления на $\left(L^{p}([-r, 0], U), U\right)$ с таким оператором управления $\beta_{U} \in$ $L\left(U,\left(L^{p}([-r, 0], U)\right)_{-1}^{Q_{U}}\right)$, что $e^{\lambda \cdot}=\left(\lambda-\left(Q_{U}\right)_{-1}\right) \beta_{U}$. Здесь оператор $e^{\lambda \cdot}: U \rightarrow$ $L^{p}([-r, 0], U)$ удовлетворяет условию $\left(e^{\lambda \cdot} x\right)(t)=e^{\lambda t} x, x \in U, t \in[-r, 0]$.

2. Основные результаты. Пусть $U=L^{1}\left(\mathbb{R}^{+}\right)$. Определим оператор $A_{m}$ формулой $\left(A_{m} f\right)(a):=-f^{\prime}(a)-\mu(a) f(a)$ для почти всех положительных $a$, причем $D\left(A_{m}\right):=W^{1,1}\left(\mathbb{R}^{+}\right)$. Введем оператор $P: D\left(A_{m}\right) \rightarrow \mathbb{C}$, действующий следующим образом: $P f:=f(0)$. Определим функцию $\xi: \mathbb{R}^{+} \rightarrow U$ формулой $\xi(t)=x(t, \cdot)$, а $\xi_{t}:[-r, 0] \rightarrow U-$ формулой $\xi_{t}(\sigma)=: \xi(t+\sigma), \sigma \in[-r, 0]$. Положим $\int_{0}^{\infty} \int_{-r}^{0} \beta(\sigma, a) x(t+\sigma, a) d \sigma d a:=\mathscr{K} \xi_{t}, t \geqslant 0$, где $\mathscr{K}: L^{1}\left([-r, 0] \times \mathbb{R}^{+}\right)$ $\rightarrow \mathbb{R}$. Очевидно, что $E:=L^{p}([-r, 0], U)$ - банахово пространство, а оператор $\mathscr{K} \in L(E, \mathbb{C})$ ограничен. В этих обозначениях задача $(1)$ принимает вид

$$
\left\{\begin{array}{l}
\frac{d \xi(t)}{d t}=A_{m} \xi(t)+L \xi_{t}, \quad P \xi(t)=\mathscr{K} \xi_{t}, \quad t \geqslant 0, \\
\xi(0)=f, \xi_{0}=F,
\end{array}\right.
$$

где $L=-\alpha(\cdot) \delta_{-r}-$ ограниченный линейный оператор из $W^{1, p}([-r, 0], U)$ в $U$, $f \in U, F \in E$. По аналогии с работой [6] преобразуем (2) в абстрактную задачу Коши на $\mathscr{E}=E \times U$

$$
\left\{\begin{array}{l}
\mathscr{X}^{\prime}(t)=\mathscr{A}_{L} \mathscr{X}, \quad t \geqslant 0, \\
\mathscr{X}(0)=\mathscr{X}_{0} .
\end{array}\right.
$$

Здесь

$$
\begin{gathered}
\mathscr{A}_{L}:=\left(\begin{array}{cc}
G_{m} & 0 \\
L & A_{m}
\end{array}\right), \quad D\left(\mathscr{A}_{L}\right)=\left\{\left(\begin{array}{c}
F \\
f
\end{array}\right) \in D\left(G_{m}\right) \times D\left(A_{m}\right): Q F=f, P f=\mathscr{K} F\right\}, \\
\left(G_{m} F\right)(\sigma, a)=\frac{\partial}{\partial \sigma} F(\sigma, a), \quad D\left(G_{m}\right)=W^{1,1}([-r, 0], U),
\end{gathered}
$$

a $Q: D\left(G_{m}\right) \rightarrow X$ определен формулой $Q F=F(0, \cdot)$. В частности, при $L=0$ мы будем использовать обозначение $\mathscr{A}_{0}$. Функция $\mathscr{X}: \mathbb{R}^{+} \rightarrow \mathscr{E}$ задана формулой $\mathscr{X}(t)=\left(\begin{array}{c}\xi_{t} \\ \xi(t)\end{array}\right)$. 
Для получения основных результатов воспользуемся теоремой об инвариантности допустимости относительно перекрестных возмущений, доказанной в нашей недавней работе [5].

Теорема 1. Предположим, что $(A, \Delta A, C)$ порождает регулярную линейную систему на $(X, X, Y)$. Тогда $\left(\left.\left(A_{-1}+\Delta A\right)\right|_{X}, C_{\Lambda}^{A}\right)$ порождает абстрактную линейную систему наблюдения, причем $C_{\Lambda}^{\left.\left(A_{-1}+\Delta A\right)\right|_{X}}=C_{\Lambda}^{A}$. Здесъ $\left(A_{-1}+\right.$ $\Delta A)\left.\right|_{X}$ - сужение оператора $A_{-1}+\Delta A$ на $X$.

Теорема 2. Оператор $\left(\mathscr{A}_{L}, D\left(\mathscr{A}_{L}\right)\right)$ порождает $C_{0}$-полугруппу на $\mathscr{E}$.

Доказательство. Введем следующие обозначения:

$$
\left.\mathscr{A}=\left(\begin{array}{cc}
G_{0} & 0 \\
0 & A_{0}
\end{array}\right), \quad \mathscr{L}=\left(\begin{array}{cc}
0 & 0 \\
L & 0
\end{array}\right), \quad \mathscr{B}=\left(\left(\begin{array}{cc}
G_{0} & 0 \\
0 & A_{0}
\end{array}\right)\right)_{-1}\left(\begin{array}{cc}
0 & K_{0} \\
L_{0} & 0
\end{array}\right)\right)\left.\right|_{\mathscr{E}} .
$$

Здесь $\left(A_{0}, D\left(A_{0}\right)\right)$ - сужение оператора $A_{m}$ на ядро оператора $P$ (указанное сужение порождает $C_{0}$-полугруппу $\left.T_{0}\right),\left(G_{0}, D\left(G_{0}\right)\right)=\left(Q_{E}, D\left(Q_{E}\right)\right)$ порождает $C_{0}$-полугруппу $S_{0}, K_{0}$ определяется формулой $K_{0}(f)=-f \chi_{[-r, 0]}, f \in V$, где $\chi_{[-r, 0]}-$ характеристическая функция отрезка $[-r, 0]$, а

$$
L_{0}(F)=-\mathscr{K}(F) e^{-\int_{0}^{\cdot} \mu(s) d s} .
$$

В силу [6] оператор $\mathscr{B}$ допустим для $\mathscr{A}$, а $\mathscr{A}_{0}=\left.\left(\mathscr{A}_{-1}+\mathscr{B}\right)\right|_{\mathscr{E}}$ порождает $C_{0}$-полугруппу. Из результатов работы [4] можно вывести, что $L$ допустим для $G_{0}=Q_{E}$. Таким образом, существуют такие постоянные $T$ и $c(T)$, что

$$
\int_{0}^{T}\left\|\left(\begin{array}{ll}
0 & 0 \\
L & 0
\end{array}\right)\left(\begin{array}{cc}
S_{0}(t) & 0 \\
0 & T_{0}(t)
\end{array}\right)\left(\begin{array}{l}
F \\
f
\end{array}\right)\right\|^{p} d t \leqslant c(T)\|F\|^{p}, \quad\left(\begin{array}{l}
F \\
f
\end{array}\right) \in D\left(\mathscr{A}_{L}\right) .
$$

Отсюда следует, что $(\mathscr{A}, \mathscr{L})$ порождает абстрактную линейную систему наблюдения.

Возьмем $g_{1} \in L^{p}([0, t], E), g_{2} \in L^{p}([0, t], X)$ и $g=\left(\begin{array}{c}g_{1} \\ g_{2}\end{array}\right)$. Можно показать, что $-\int_{0}^{t} S_{0}(t-s)\left[g_{2}(s) \chi_{[-r, 0]}\right] d s \in W^{1, p}([-r, 0], X)$ и $\left(-\int_{0}^{t} S_{0}(t-s)\left[g_{2}(s) \chi_{[-r, 0]}\right] d s\right)(0)$ $=0$, из чего следует, что $-\int_{0}^{t} S_{0}(t-s)\left[g_{2}(s) \chi_{[-r, 0]}\right] d s \in D\left(G_{0}\right)$. Далее,

$$
G_{0}\left(-\int_{0}^{t} S_{0}(t-s)\left[g_{2}(s) \chi_{[-r, 0]}\right] d s\right)(\cdot)=\left\{\begin{array}{ll}
g_{2}(\cdot+t), & \cdot+t \geqslant 0, \\
0, & \cdot+t<0
\end{array}=\left(\phi(t) g_{2}\right)(\cdot) .\right.
$$

Согласно [4], тройка $\left(Q_{E}, \beta_{E}, L\right)$ порождает регулярную линейную систему. Отсюда следует, что $\phi(t) g_{2} \in D\left(L_{\Lambda}^{G_{0}}\right)$ при п. в. $t \geqslant 0$ и $L_{\Lambda}^{G_{0}} \phi(\cdot) \in L\left(L_{\mathrm{loc}}^{p}\left(\mathbb{R}^{+}, E\right)\right.$, $\left.L_{\mathrm{loc}}^{p}\left(\mathbb{R}^{+}, X\right)\right)$. Тогда легко видеть, что $\mathscr{L}_{\Lambda}^{\mathscr{A}}=\left(\begin{array}{cc}0 & 0 \\ L_{\Lambda}^{G_{0}} & 0\end{array}\right)$, где $D\left(\mathscr{L}_{\Lambda}^{\mathscr{A}}\right)=D\left(L_{\Lambda}^{G_{0}}\right) \times X$. Применяя [6, теорема 5], получаем, что $-\int_{0}^{t} T_{0}(t-s)\left[\mathscr{K}\left(g_{1}(s)\right) e^{-\int_{0} \cdot \mu(\sigma) d \sigma}\right] d s \in$ $D\left(A_{0}\right)$. Прямое вычисление показывает, что

$$
\Phi(t) g=\left(\begin{array}{c}
G_{0}\left(-\int_{0}^{t} S_{0}(t-s)\left[g_{2}(s) \chi_{[-r, 0]}\right] d s\right) \\
A_{0}\left(-\int_{0}^{t} T_{0}(t-s)\left[\mathscr{K}\left(g_{1}(s)\right) e^{-\int_{0}^{\cdot} \mu(\sigma) d \sigma}\right] d s\right)
\end{array}\right) \in D\left(\mathscr{L}_{\Lambda}^{\mathscr{A}}\right)
$$


при почти всех неотрицательных $t$. Кроме того, существуют такие положительные постоянные $t_{0}$ и $d$, что

$$
\int_{0}^{t_{0}}\left\|\left(\begin{array}{ll}
0 & 0 \\
L & 0
\end{array}\right)_{\Lambda}^{\mathscr{A}} \Phi(t) g\right\|^{p} d t \leqslant d\|g\|^{p} .
$$

Следовательно, $\left(\mathscr{A}_{0}, \mathscr{B}, \mathscr{L}\right)$ порождает регулярную линейную систему. По теореме 1 оператор $\mathscr{L}_{\Lambda}^{\mathscr{A}}$ является допустимым для $\mathscr{A}_{0}$ и $\mathscr{L}_{\Lambda}^{\mathscr{A}_{0}}=\mathscr{L}_{\Lambda}^{\mathscr{A}}$. Таким образом, $\mathscr{L}_{\Lambda}^{\mathscr{A}_{0}}=\mathscr{L}$ на $D\left(\mathscr{A}_{0}\right)$ и $\mathscr{L}$ является допустимым для $\mathscr{A}_{0}$. Следовательно, $\mathscr{A}_{L}=\mathscr{A}_{0}+\mathscr{L}$ порождает $C_{0}$-полугруппу [3, теорема 2.1].

Аналогично тому, как это делается в [6, теорема 2], мы получаем из теоремы 2, что система (1) корректна, т. е. для любого $\left(\begin{array}{c}F \\ g\end{array}\right) \in D\left(\mathscr{A}_{L}\right)$ существует единственная функция $\xi:[-r, \infty) \rightarrow U$, непрерывно дифференцируемая на $[0, \infty)$ и такая, что $(2)$ выполняется, причем решение непрерывно зависит от начальных данных.

\section{ЛитерАТУРА}

[1] K.-J. Engel, R. Nagel, One Parameter Semigroups for Linear Evolutional Equations, Springer-Verlag, New York, 2000. [2] G. Greiner, in: Infinite-Dimensional Systems (Retzhof, 1983), Lecture Notes in Math., vol. 1076, Springer-Verlag, Berlin, 1984, 86-100. [3] S. Hadd, Semigroup Forum, 70:3 (2005), 451-465. [4] S. Hadd, A. Idrissi, A. Rhandi, Math. Control Signals Syst., 18:3 (2006), 272-291. [5] Z. D. Mei, J. G. Peng, Proc. Amer. Math. Soc., 138 (2010), 4455-4468. [6] S. Piazzera, Math. Methods Appl. Sci., 27:4 (2004), 427-439. [7] S. Piazzera, L. Tonetto, J. Evol. Equ., 5:1 (2005), 61-77. [8] H. Prüss, W. Schappacher, in: Jahrbuch Überblicke Mathematik, Vieweg, Braunschweig, 1994, 7490. [9] D. Salamon, Trans. Amer. Math. Soc., 300:2 (1987), 383-431. [10] G. Weiss, SIAM J. Control Optimization, 27:3 (1989), 527-545. [11] G. Weiss, Israel J. Math., 65:1 (1989), 17-43. [12] G. Weiss, Trans. Amer. Math. Soc., 342:2 (1994), 827-854.

School of Mathematics and Statistics,

Xi'an Jiaotong University, China

Поступила в редакцию e-mail: zhdmei@mail.xjtu.edu.cn 7 декабря 2012 г.

School of Mathematics and Statistics,

Xi'an Jiaotong University, China

e-mail: jgpeng@mail.xjtu.edu.cn

Заведующая редакцией и научный редактор Г. М. Цукерман

Подписано к печати 24.04.2015. Дата выхода в свет 20.05.2015. Формат 70×100/16 Печать цифровая. Усл. печ. л. 7,8. Усл. кр.-отт. 1,7 тыс. Бум. л. 3,0 Уч.-изд. л. 8,0. Тираж 160 экз. Заказ 121. 